\title{
ON KÄHLER STRUCTURES OVER SYMMETRIC PRODUCTS OF A RIEMANN SURFACE
}

\author{
INDRANIL BISWAS
}

(Communicated by Varghese Mathai)

\begin{abstract}
Given a positive integer $n$ and a compact connected Riemann surface $X$, we prove that the symmetric product $S^{n}(X)$ admits a Kähler form of nonnegative holomorphic bisectional curvature if and only if genus $(X) \leq 1$. If $n$ is greater than or equal to the gonality of $X$, we prove that $S^{n}(X)$ does not admit any Kähler form of nonpositive holomorphic sectional curvature. In particular, if $X$ is hyperelliptic, then $S^{n}(X)$ admits a Kähler form of nonpositive holomorphic sectional curvature if and only if $n=1 \leq \operatorname{genus}(X)$.
\end{abstract}

\section{INTRODUCTION}

This work was inspired by $[\mathrm{BR}$. The following is the main theorem proved there (see $[\mathrm{BR}$, Theorem 1.1]):

Let $X$ be a compact connected Riemann surface of genus at least two. If

$$
n \leq 2(\operatorname{genus}(X)-1),
$$

then the symmetric product $S^{n}(X)$ does not admit any Kähler form of nonnegative holomorphic bisectional curvature.

By nonnegative holomorphic bisectional curvature we mean that all holomorphic bisectional curvatures are nonnegative. Similarly, by nonpositive holomorphic sectional curvature we mean that all holomorphic sectional curvatures are nonpositive.

Our aim here is to settle the cases not considered in $[\mathrm{BR}$. We prove the following theorem (see Theorem 2.3):

Theorem 1.1. Let $X$ be a compact connected Riemann surface, and let $n$ be a fixed positive integer. The symmetric product $S^{n}(X)$ admits a Kähler form of nonnegative holomorphic bisectional curvature if and only if genus $(X) \leq 1$.

The gonality of a compact connected Riemann surface $X$ is the smallest integer $d$ such that there is a nonconstant holomorphic map $X \longrightarrow \mathbb{C P}^{1}$ of degree $d$.

We prove the following (see Proposition 3.2 ):

Proposition 1.2. Let $X$ be a compact connected Riemann surface. Let $d$ be the gonality of $X$. Take any integer $n \geq d$. Then $S^{n}(X)$ does not admit any Kähler form of nonpositive holomorphic sectional curvature.

Note that a Kähler form of nonpositive holomorphic bisectional curvature is also a Kähler form of nonpositive holomorphic sectional curvature.

Received by the editors August 25, 2011.

2010 Mathematics Subject Classification. Primary 14C20, 32Q05, 32Q10.

Key words and phrases. Riemann surface, symmetric product, Kähler form, curvature. 
Proposition 1.2 gives the following (see Corollary [3.3):

Corollary 1.3. Let $X$ be a hyperelliptic Riemann surface. The compact complex manifold $S^{n}(X)$ admits a Kähler form of nonpositive holomorphic sectional curvature if and only if $n=1 \leq \operatorname{genus}(X)$.

\section{Nonnegative holomorphic Bisectional CURVATURe}

Let $X$ be a compact connected Riemann surface. The genus of $X$ will be denoted by $g$. For any positive integer $n$, let $S^{n}(X)$ be the symmetric product of $X$. Therefore, $S^{n}(X)$ is the quotient of the Cartesian product $X^{n}$ by the natural action of the group of permutations of the index set $\{1, \ldots, n\}$. It is known that $S^{n}(X)$ is a smooth complex projective variety of dimension $n$.

In this section we address the question whether $S^{n}(X)$ admits a Kähler structure of nonnegative holomorphic bisectional curvature.

Proposition 2.1. Assume that $g \geq 2$. The complex manifold $S^{n}(X)$ does not admit any Kähler metric of nonnegative holomorphic bisectional curvature.

Proof. If $n \leq 2 g-2$, then this is proved in [BR] (see [BR, Theorem 1.1]). So we assume that $n>2 g-2$.

Let $J^{n}(X)$ be the Picard variety parametrizing isomorphism classes of holomorphic line bundles over $X$ of degree $n$. Fix a Poincaré line bundle

$$
\mathcal{L} \longrightarrow X \times J^{n}(X)
$$

(see [ACGH, Ch. IV,$\S 2]$ for the construction of a Poincaré line bundle). Let

$$
p: X \times J^{n}(X) \longrightarrow J^{n}(X)
$$

be the projection to the second factor. Since $n>2 g-2$, we have $\operatorname{degree}\left(L^{*} \otimes\right.$ $\left.K_{X}\right)<0$ for any line bundle $L$ of degree $n$ on $X$, where $K_{X}$ is the holomorphic cotangent bundle of $X$. Hence by Serre duality, we have

$$
H^{1}(X, L)=H^{0}\left(X, L^{*} \otimes K_{X}\right)^{*}=0
$$

for any holomorphic line bundle $L$ of degree $n$. Therefore, for the projection $p$ in (2.2), we have

$$
R^{1} p_{*} \mathcal{L}=0
$$

and also

$$
\mathcal{V}:=p_{*} \mathcal{L} \longrightarrow J^{n}(X)
$$

is a holomorphic vector bundle of rank $n-g+1$.

Consider the projective bundle

$$
f: P(\mathcal{V}) \longrightarrow J^{n}(X)
$$

parametrizing the lines in the fibers of the vector bundle $\mathcal{V}$ in (2.3). Since any two Poincaré line bundles over $X \times J^{n}(X)$ differ by tensoring with a line bundle pulled back from $J^{n}(X)$ ACGH, p. 166], using the projection formula we conclude that the projective bundle $P(\mathcal{V})$ is actually independent of the choice of the Poincare line bundle $\mathcal{L}$.

The total space of $P(\mathcal{V})$ is identified with $S^{n}(X)$. Points of $P(\mathcal{V})$ parametrize isomorphism classes of pairs of the form $(L, s)$, where $L \in J^{n}(X)$ and $s$ is a holomorphic section of $L$ which is not identically zero. The identification of $P(\mathcal{V})$ with $S^{n}(X)$ sends any pair $(L, s)$ to the divisor of the section $s$. 
Assume that $S^{n}(X)$ has a Kähler metric of nonnegative holomorphic bisectional curvature. Therefore, the total space of $P(\mathcal{V})$ has a Kähler metric of nonnegative holomorphic bisectional curvature. This implies that the anticanonical line bundle $K_{P(\mathcal{V})}^{-1}:=\bigwedge^{n} T P(\mathcal{V})$ is numerically effective.

Let $T_{\text {rel }} \subset T P(\mathcal{V})$ be the relative tangent bundle for the projection $f$ in (2.4). In other words, $T_{\text {rel }}$ is the kernel of the differential $d f: T P(\mathcal{V}) \longrightarrow f^{*} T J^{n}(X)$. Since the tangent bundle $T J^{n}(X)$ is trivial, the holomorphic line bundle

$$
\operatorname{det}\left(T_{\text {rel }}\right):=\bigwedge^{n-g} T_{\text {rel }} \longrightarrow P(\mathcal{V})
$$

is identified with $K_{P(\mathcal{V})}^{-1}$. It was noted above that $K_{P(\mathcal{V})}^{-1}$ is numerically effective. Therefore, we now conclude that the line bundle $\operatorname{det}\left(T_{\text {rel }}\right)$ in (2.5) is numerically effective.

Since $\operatorname{det}\left(T_{\text {rel }}\right)$ is numerically effective, the vector bundle $\mathcal{V}$ has the property that

$$
c_{2}(\operatorname{ad}(\mathcal{V}))=0
$$

[BB, Theorem 1.1], where $\operatorname{ad}(\mathcal{V}) \subset \operatorname{End}(\mathcal{V})=\mathcal{V} \otimes \mathcal{V}^{*}$ is the subbundle of co-rank one defined by the sheaf of endomorphisms of trace zero.

For a particular choice of the Poincaré bundle $\mathcal{L}$,

$$
\operatorname{ch}(\mathcal{V})=(n-g+1)-\theta,
$$

where $\theta \in H^{2}\left(J^{n}(X), \mathbb{Q}\right)$ is the class of a theta divisor; see lines 2-4 (from top) of [ACGH, p. 336]. From (2.7) it follows immediately that

$$
c_{2}(\operatorname{ad}(\mathcal{V}))=-c h_{2}(\operatorname{ad}(\mathcal{V}))=\theta^{2} .
$$

Note that $\theta^{2} \neq 0$ because $\theta$ is an ample class and $\operatorname{dim} J^{n}(X) \geq 2$. But this contradicts (2.6). Therefore, we conclude that $S^{n}(X)$ does not admit any Kähler metric of nonnegative holomorphic bisectional curvature.

Proposition 2.2. Assume that $g \leq 1$. The complex manifold $S^{n}(X)$ admits a Kähler metric of nonnegative holomorphic bisectional curvature.

Proof. First assume that $g=0$. Then $S^{n}(X)$ is biholomorphic to $\mathbb{C P}^{n}$. A FubiniStudy metric on $\mathbb{C P}^{n}$ has positive holomorphic bisectional curvature. (In fact this property of existence of a Kähler form of positive holomorphic bisectional curvature characterizes $\mathbb{C P}^{n}[\mathrm{Mo}$, [SY].)

Now assume that $g=1$. Consider the vector bundle $\mathcal{V} \longrightarrow J^{n}(X)$ constructed as in (2.3). As before, we have

$$
P(\mathcal{V})=S^{n}(X)
$$

We will show that the projective bundle $P(\mathcal{V})$ over $J^{n}(X)$ is given by a representation of $\pi_{1}\left(J^{n}(X), x_{0}\right)$ in the projective unitary group $\mathrm{PU}(n)$.

To prove this, first note that a theorem due to Ein-Lazarsfeld and Kempf says that the vector bundle $\mathcal{V}$ on $J^{n}(X)$ is stable [EL, p. 149, Theorem], Ke, p. 285, Theorem 3]. Since $\operatorname{dim} J^{n}(X)=1$, any stable vector bundle on $J^{n}(X)$ has a projectively flat unitary connection [NS]; this also follows from Atiyah's classification of vector bundles over an elliptic curve $\mathrm{At}$. This proves the above statement that $P(\mathcal{V})$ is given by a representation of $\pi_{1}\left(J^{n}(X), x_{0}\right)$ in $\mathrm{PU}(n)$. In fact, Kempf showed that the natural $L^{2}$-metric on $\mathcal{V}$ is projectively flat [Ke, p. 286, Corollary 6].

Fix a representation

$$
\rho: \pi_{1}\left(J^{n}(X), x_{0}\right) \longrightarrow \mathrm{PU}(n)
$$


corresponding to a projectively flat unitary connection on $P(\mathcal{V})$. Let $\widetilde{J^{n}(X)} \longrightarrow$ $J^{n}(X)$ be the universal cover associated to the base point $x_{0}$; note that $\widehat{J^{n}(X)}$ is a principal $\pi_{1}\left(J^{n}(X), x_{0}\right)$-bundle over $J^{n}(X)$. Let

$$
\varphi: F_{\rho}:=\widehat{J^{n}(X)} \times{ }^{\rho} \mathbb{C P}^{n-1} \longrightarrow J^{n}(X)
$$

be the holomorphic fiber bundle associated to the principal $\pi_{1}\left(J^{n}(X), x_{0}\right)$-bundle

$$
\widetilde{J^{n}(X)} \longrightarrow J^{n}(X)
$$

for the action of $\pi_{1}\left(J^{n}(X), x_{0}\right)$ on $\mathbb{C P}^{n-1}$ defined by $\rho$ and the standard action of $\mathrm{PU}(n)$ on $\mathbb{C P}^{n-1}$. The holomorphic fiber bundle $F_{\rho} \stackrel{\varphi}{\longrightarrow} J^{n}(X)$ is equipped with a flat holomorphic connection; this connection will be denoted by $\nabla$. The total space of $P(\mathcal{V})$ is biholomorphic to the total space of $F_{\rho}$ because both the fiber bundles are given by $\rho$.

Fix a Fubini-Study metric $\omega_{F S}$ on $\mathbb{C P}^{n-1}$ preserved by the standard action of $\mathrm{PU}(n)$ on $\mathbb{C P}^{n-1}$. This metric defines a closed $(1,1)$-form $\omega_{F}$ on the total space of $F_{\rho}$ which is Kähler on the fibers of the projection $\varphi$ in (2.8); this form $\omega_{F}$ is uniquely determined by the following conditions:

(1) for any point $z \in F_{\rho}$ and any horizontal tangent vector $v \in T_{z}^{1,0} F_{\rho} \oplus T_{z}^{0,1} F_{\rho}$ for the above connection $\nabla$,

$$
i_{v} \omega_{F}(z)=0
$$

(it is the contraction of $\omega_{F}(z) \in \Omega_{z}^{1,1} F_{\rho}$ by $v$ ), and

(2) the restriction of $\omega_{F}$ to each fiber of the projection $F_{\rho} \longrightarrow J^{n}(X)$ is $\omega_{F S}$.

The closed $(1,1)$-form $\omega_{F}$ can also be described as follows. Consider the $(1,1)-$ form $p_{\mathbb{C P} n-1}^{*} \omega_{F S}$ on $\widehat{J^{n}(X)} \times \mathbb{C P}^{n-1}$, where $p_{\mathbb{C P}^{n-1}}$ is the projection of $\widehat{J^{n}(X)} \times$ $\mathbb{C P P}^{n-1}$ to $\mathbb{C P}^{n-1}$. This form $p_{\mathbb{C P} n-1}^{*} \omega_{F S}$ descends to the quotient space $\widetilde{J^{n}(X)} \times^{\rho}$ $\mathbb{C P}^{n-1}$. This descended form coincides with $\omega_{F}$.

For any Kähler form $\omega_{0}$ on $J^{n}(X)$, it is straightforward to check that

$$
\omega_{F}+\varphi^{*} \omega_{0}
$$

is a Kähler form on the total space of $F_{\rho}$, where $\varphi$ is the projection in (2.8).

Since $J^{n}(X)$ is an elliptic curve, it has a Kähler metric $\omega_{J^{n}(X)}$ of curvature zero (take any translation invariant metric on $J^{n}(X)$ ).

Since the Fubini-Study metric $\omega_{F S}$ has positive holomorphic bisectional curvature, and the curvature of $\omega_{J^{n}(X)}$ is zero, we conclude that the holomorphic bisectional curvature of the Kähler form

$$
\omega_{F}+\varphi^{*} \omega_{J^{n}(X)}
$$

on the total space of $F_{\rho}$ is nonnegative. Indeed, this follows immediately from the fact that the Kähler form $\omega_{F}+\varphi^{*} \omega_{J^{n}(X)}$ is locally the product of $\omega_{F S}$ on $\mathbb{C P}^{n-1}$ and $\omega_{J^{n}(X)}$ on $J^{n}(X)$. Since the total space of $F_{\rho}$ is biholomorphic to the total space of $P(\mathcal{V})$, the proof of the proposition is complete.

Combining Proposition 2.1 and Proposition 2.2, we have the following theorem:

Theorem 2.3. Let $X$ be a compact connected Riemann surface, and let $n$ be a fixed positive integer. The compact complex manifold $S^{n}(X)$ admits a Kähler form of nonnegative holomorphic bisectional curvature if and only if genus $(X) \leq 1$. 


\section{Nonpositive holomorphic SECtional CURVATURE}

We begin by recalling a property of compact Kähler manifolds with nonpositive holomorphic sectional curvature.

Lemma 3.1. Let $M$ be a compact connected Kähler manifold admitting a Kähler form of nonpositive holomorphic sectional curvature. Then there is no nonconstant holomorphic map from $\mathbb{C P}^{1}$ to $M$.

Proof. See Gr, p. 40, Corollary 4.5] for a proof of this lemma. In Gr, "negatively curved" Kähler metric means one with nonpositive holomorphic sectional curvature (see Definition in [Gr, p. 39]).

Let $X$ be a compact connected Riemann surface. The gonality of $X$ is the smallest integer $d$ such that there is a nonconstant holomorphic map $X \longrightarrow \mathbb{C P}^{1}$ of degree $d$. Equivalently, gonality of $X$ is the smallest integer $d$ such that there is a holomorphic line bundle $\xi$ on $X$ of degree $d$ with $\operatorname{dim} H^{0}(X, \xi) \geq 2$. Therefore, from the Riemann-Roch theorem it follows that the gonality of $X$ is bounded above by $\operatorname{genus}(X)+1$.

If the gonality of $X$ is two, then $X$ is called a hyperelliptic Riemann surface.

Proposition 3.2. Let $X$ be a compact connected Riemann surface of gonality $d$. If $n \geq d$, then $S^{n}(X)$ does not admit any Kähler form of nonpositive holomorphic sectional curvature. In particular, $S^{n}(X)$ does not admit a Kähler form of nonpositive holomorphic sectional curvature if $n>\operatorname{genus}(X)$.

Proof. Let $\phi: X \longrightarrow \mathbb{C P}^{1}$ be a nonconstant holomorphic map of degree $\delta$. Then we get a holomorphic map

$$
\widetilde{\phi}: \mathbb{C P}^{1} \longrightarrow S^{\delta}(X)
$$

that sends any $x \in \mathbb{C P}^{1}$ to the scheme-theoretic inverse image $\phi^{-1}(x)$ of $x$. This map $\widetilde{\phi}$ is clearly nonconstant.

If

$$
\psi: \mathbb{C P}^{1} \longrightarrow S^{a}(X)
$$

is a nonconstant holomorphic map, and $b>a$, then we can construct a nonconstant holomorphic map

$$
\mathbb{C P}^{1} \longrightarrow S^{b}(X)
$$

as follows: fix a point $y_{0} \in X$, and send any $x \in \mathbb{C P}^{1}$ to $\psi(x)+(b-a) y_{0} \in S^{b}(X)$.

In view of these two observations, the proposition follows from Lemma 3.1

Corollary 3.3. Let $X$ be a hyperelliptic Riemann surface. The compact complex manifold $S^{n}(X)$ admits a Kähler form of nonpositive holomorphic sectional curvature if and only if $n=1$.

Proof. Since $X$ is hyperelliptic, we have genus $(X)>0$; hence $X=S^{1}(X)$ admits a Kähler form of nonpositive holomorphic sectional curvature. If $n \geq 2$, from Proposition 3.2 we know that $S^{n}(X)$ does not admit any Kähler form of nonpositive holomorphic sectional curvature. 


\section{REFERENCES}

[ACGH] E. Arbarello, M. Cornalba, P. A. Griffiths and J. Harris, Geometry of algebraic curves. Vol. I, Grundlehren der Mathematischen Wissenschaften, 267, Springer-Verlag, New York, 1985. MR770932(86h:14019)

[At] M. F. Atiyah, Vector bundles over an elliptic curve, Proc. London Math. Soc. 7 (1957), 414-452. MR0131423 (24:A1274)

[BB] I. Biswas and U. Bruzzo, On semistable principal bundles over a complex projective manifold, Int. Math. Res. Not. (2008), Art. ID rnn035. MR2426752 (2009k:14077)

[BR] M. Bökstedt and N. M. Romão, On the curvature of vortex moduli spaces, http://arxiv.org/abs/1010.1488.

[EL] L. Ein and R. K. Lazarsfeld, Stability and restrictions of Picard bundles, with an application to the normal bundles of elliptic curves, in: Complex projective geometry (Trieste, 1989/Bergen, 1989), 149-156, London Math. Soc. Lecture Note Ser., 179, Cambridge Univ. Press, Cambridge, 1992. MR.1201380 (94a:14015)

[Gr] P. A. Griffiths, Two theorems on extensions of holomorphic mappings, Invent. Math. 14 (1971), 27-62. MR0293123 (45:2202)

[Ke] G. Kempf, A problem of Narasimhan, in: Curves, Jacobians, and abelian varieties (Amherst, MA, 1990), 283-286, Contemp. Math., 136, Amer. Math. Soc., Providence, RI, 1992. MR.1188202 (94e:14043)

[Mo] S. Mori, Projective manifolds with ample tangent bundles, Ann. of Math. (2) 110 (1979), 593-606. MR554387 (81j:14010)

[NS] M. S. Narasimhan and C. S. Seshadri, Stable and unitary vector bundles on a compact Riemann surface, Ann. of Math. (2) 82 (1965), 540-567. MR0184252 (32:1725)

[SY] Y.-T. Siu and S.-T. Yau, Compact Kähler manifolds of positive bisectional curvature, Invent. Math. 59 (1980), 189-204. MR.577360 (81h:58029)

School of Mathematics, Tata Institute of Fundamental Research, Homi Bhabha ROAD, BOMBAY 400005, INDIA

E-mail address: indranil@math.tifr.res.in 